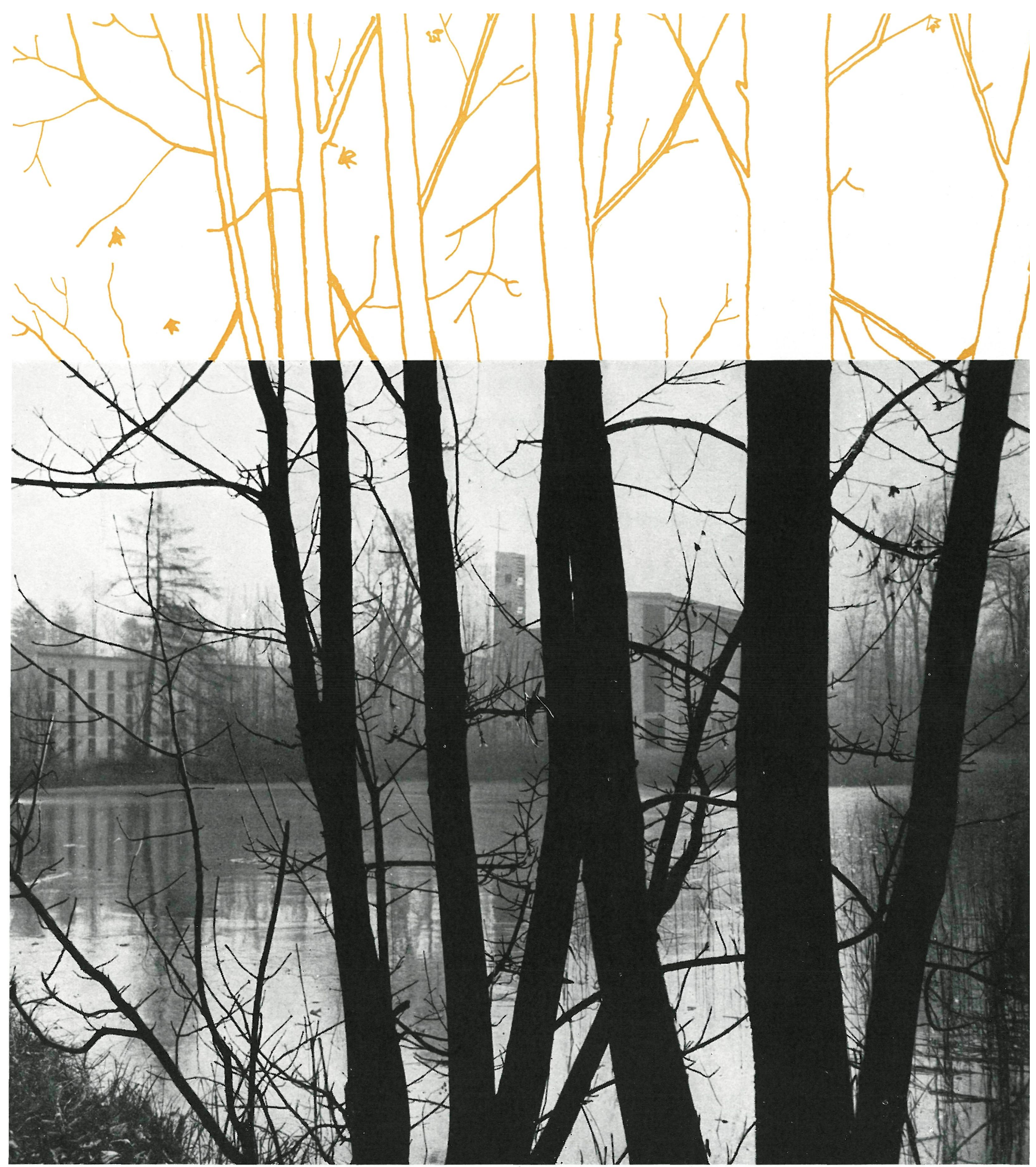

$148 \cdot 56$

\title{
convento franciscano en ORSAY
}

LUC y XAVIER ARSENE-HENRY y E. BESNARD-BERNADAC, arquifectos 


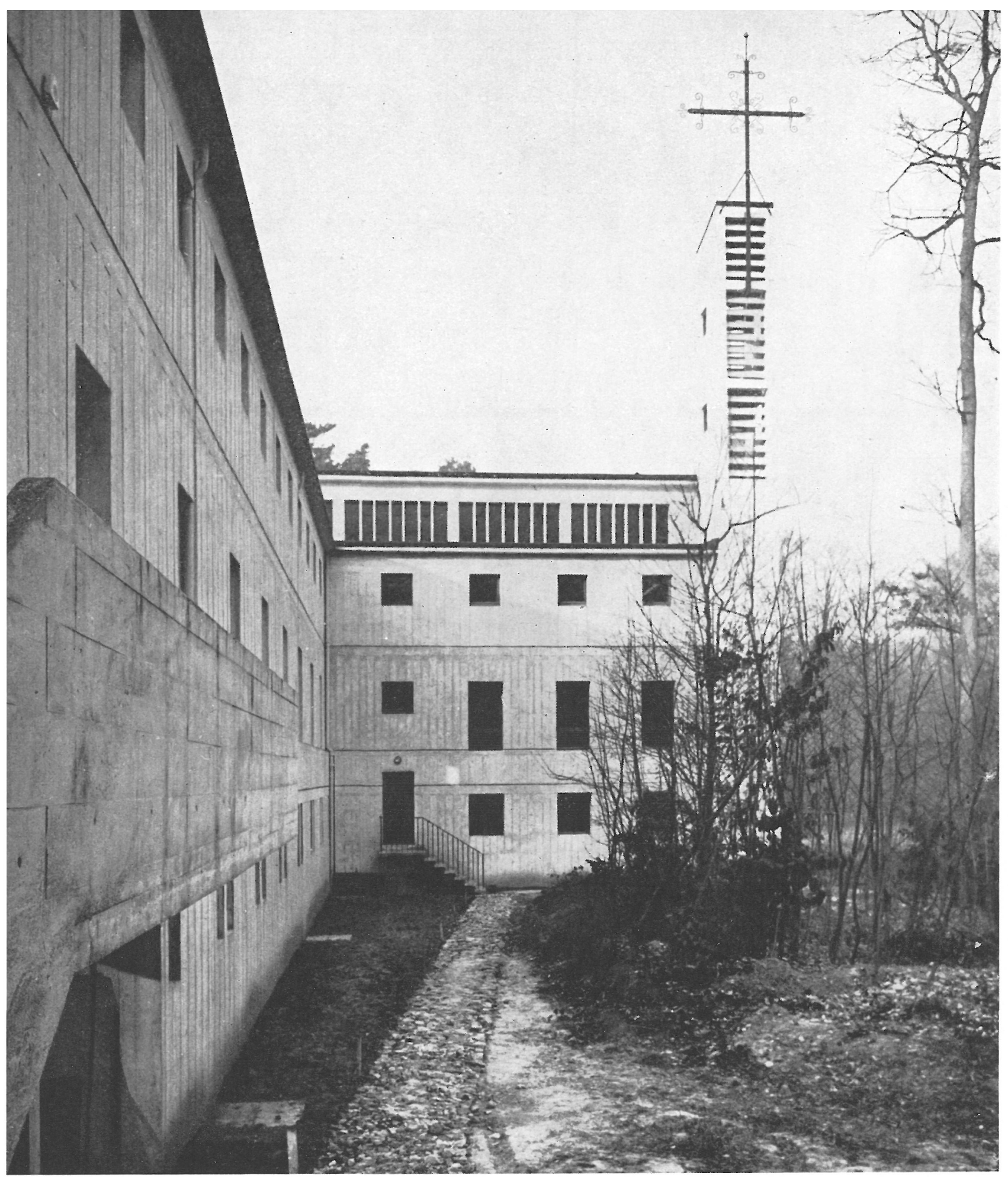

Este es el primer conjunto monástico completo y totalmente equipado que se construye en Francia desde el siglo XVIII. Desde el punto de vista religioso representa gran interés, ya que significa la posibilidad de adaptarse, tanto en el interior como en el exterior, a las necesidades y exigencias de la Orden, situándonos siempre en el momento actual constructivo, estético y de comodidades toleradas por la regla.

El convento, situado en una zona de bosque y a media hora de la Sorbona y del Instituto Católico, se destina a centro de teología o noviciado de los frailes franciscanos. El terreno ofrece un claro declive hacia una pequeña laguna, lo cual ha permitido distribuir los locales de la comunidad en un nivel intermedio.

El programa presentado a los arquitectos puede extractarse en los siguientes conceptos: "El marco en que han de vivir los religiosos será austero y desnudo. Deben preocuparse, sin perjudicar a la limpieza y solidez, de dejar desnudos o vistos, tanto en el exterior como en el interior, los materiales necesarios para la construcción. 


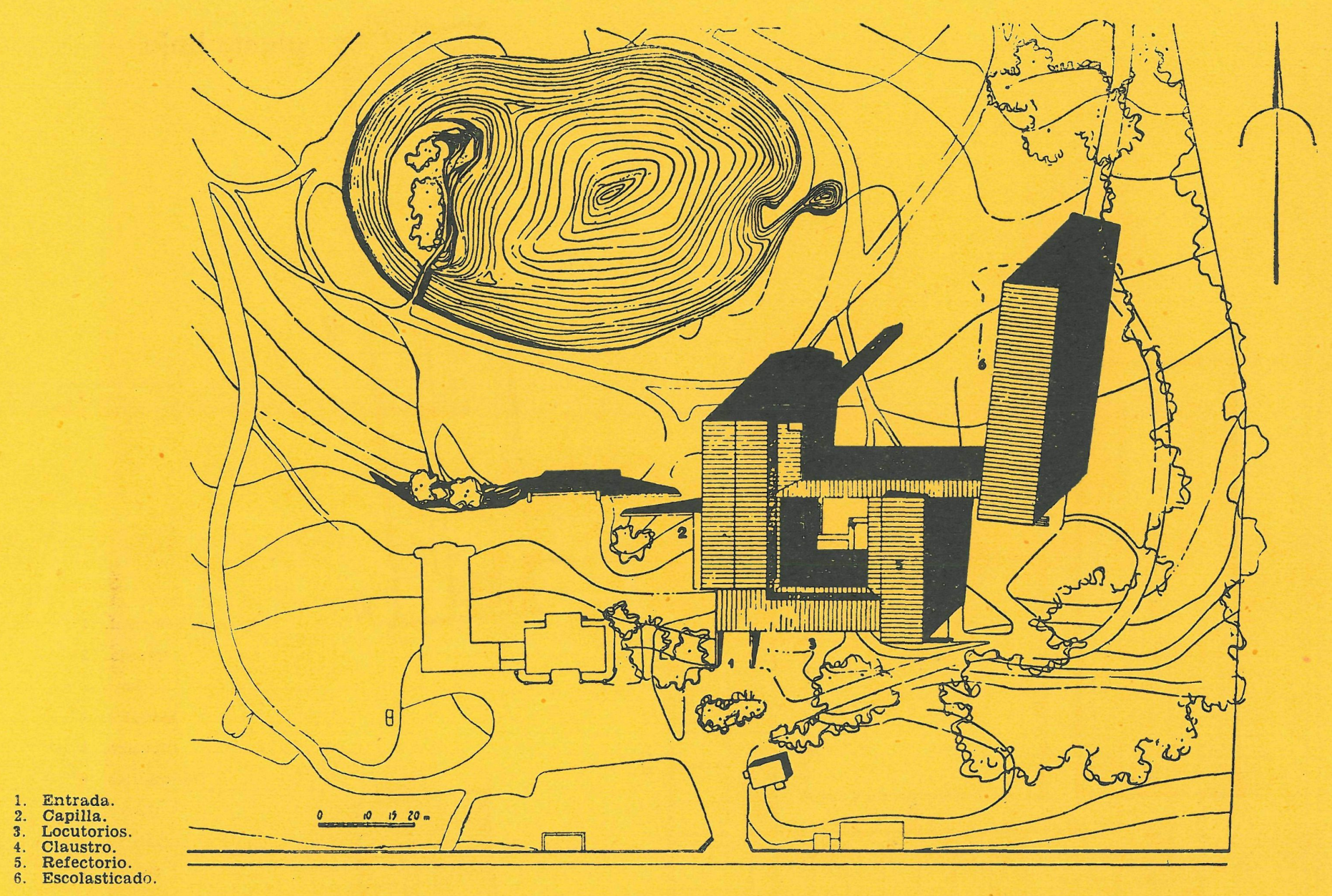

Asimismo, cuidarán de combinar la austeridad, la pobreza y la sinceridad con la luminosidad y el respeto al sol, al aire y a la naturaleza. No deberán olvidar, en ningún caso, que esta construcción se hace gracias al fruto y que las privaciones de muchísima gente, humilde en su mayoría $y$, por lo tanto, nada debe derrocharse. La conservación, en fin, del edificio, será lo menos costosa posible."

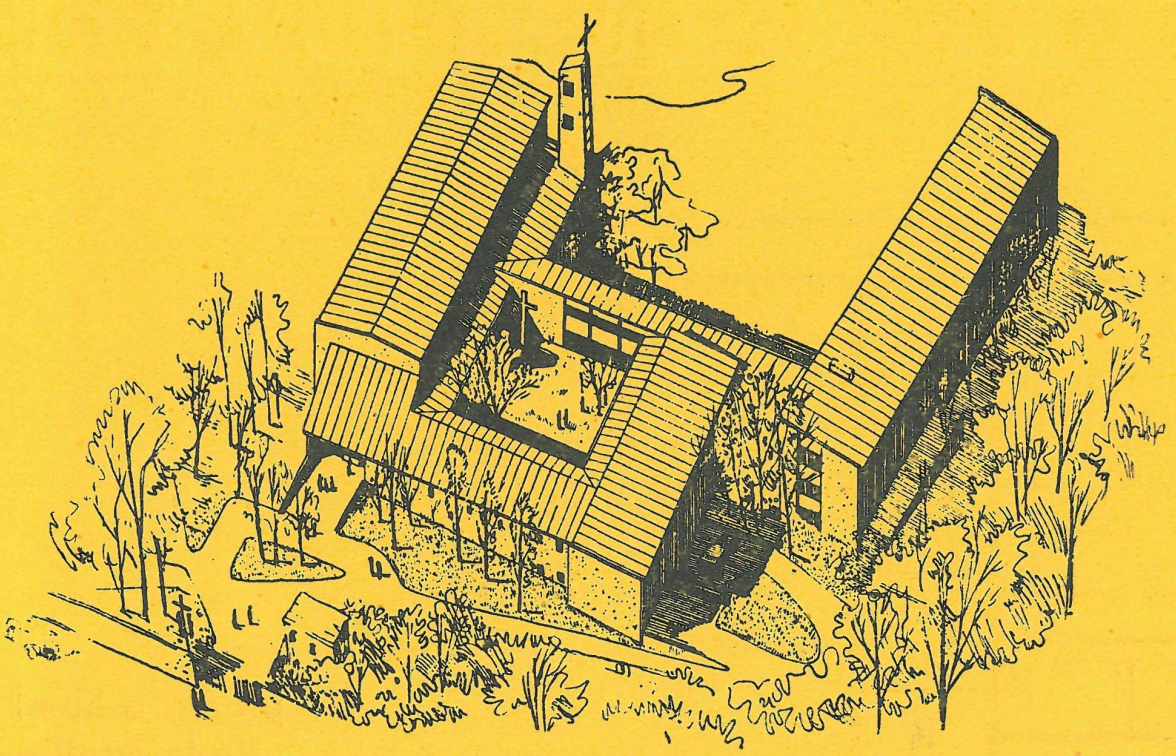

Estas premisas dictadas por la propiedad, forzaron la solución adoptada. El convento está construído en su totalidad con hormigon: se trata, pleta e importante, destinada a dar a este material, por sí mismo, un valor estético, utimismo, un valor estético, utiadaptabilidad de forma y textura de este material. La cimentación se ha realizado con pilotes prefabricados "in situ". Los muros son de hormigón de cemento blanco; sus superficies adquieren forma, vida y textura gracias al ma, vida y textura gracias al tamente cuidados, hasta el extremo de presentar un estriado que tiende a evitar las desagradables apariencias de las juntas de hormigonado. El aislamiento conveniente se consigue mediante cámaras de aire. 


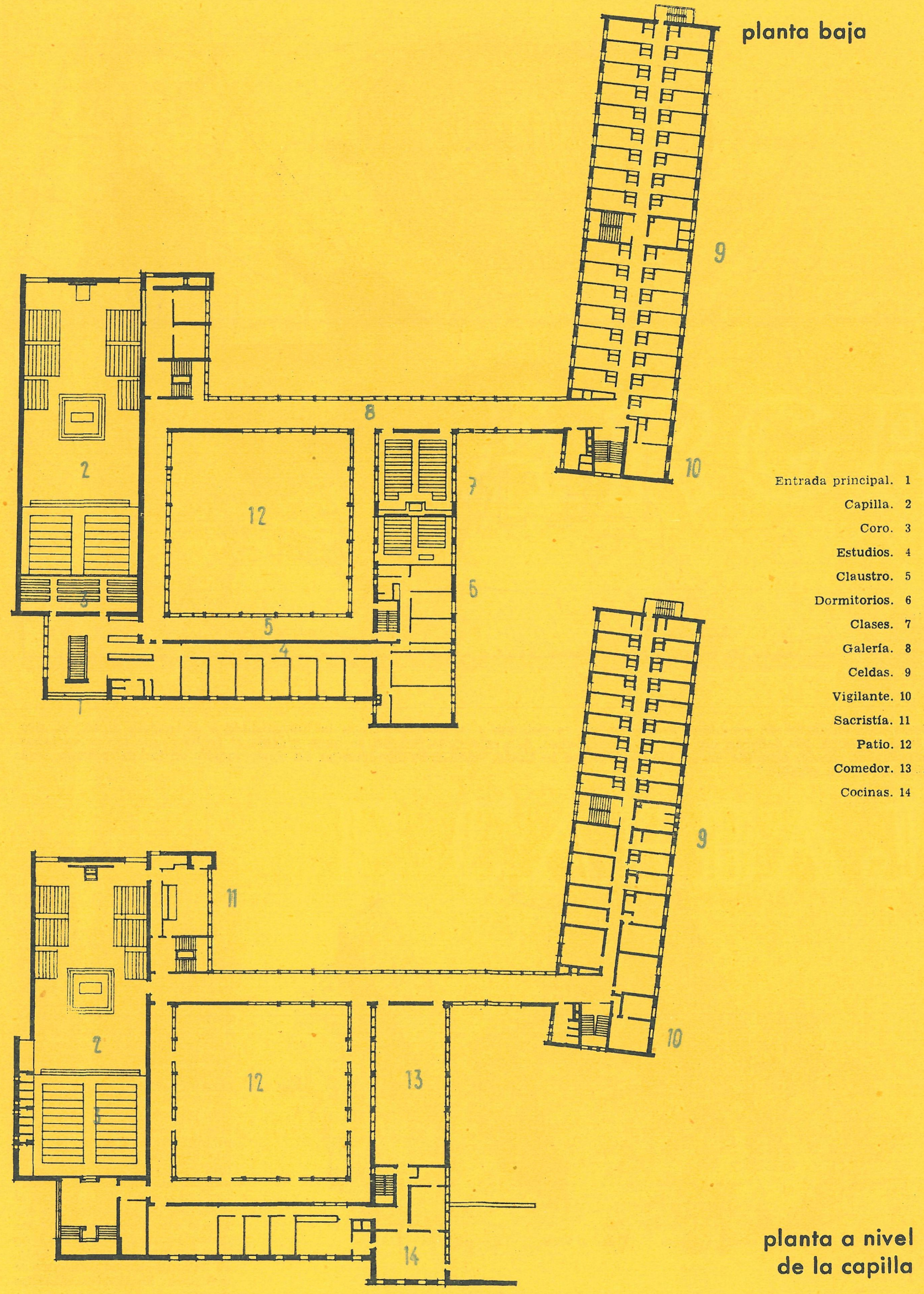



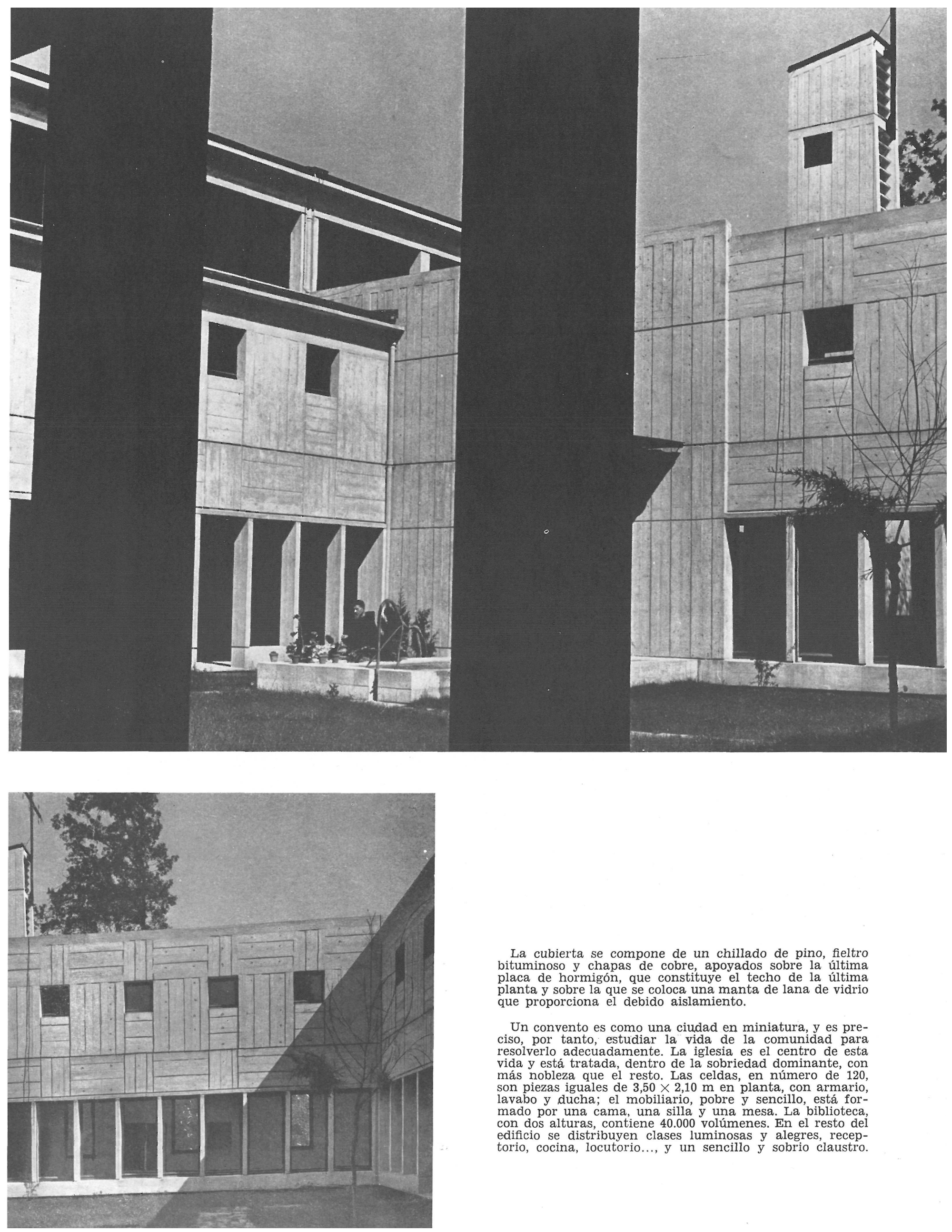

La cubierta se compone de un chillado de pino, fieltro bituminoso y chapas de cobre, apoyados sobre la última placa de hormigón, que constituye el techo de la última planta y sobre la que se coloca una manta de lana de vidrio que proporciona el debido aislamiento.

Un convento es como una ciudad en miniatura, y es preciso, por tanto estudiar la vida de la comunidad para resolverlo adecuadamente. La iglesia es el centro de esta vida y está tratada, dentro de la sobriedad dominante, con más nobleza que el resto. Las celdas, en número de 120, son piezas iguales de $3,50 \times 2,10 \mathrm{~m}$ en planta, con armario, lavabo y ducha; el mobiliario, pobre y sencillo, está formado por una cama, una silla y una mesa. La biblioteca, con dos alturas, contiene 40.000 volúmenes. En el resto del edificio se distribuyen clases luminosas y alegres, receptorio, cocina, locutorio..., y un sencillo y sobrio claustro. 

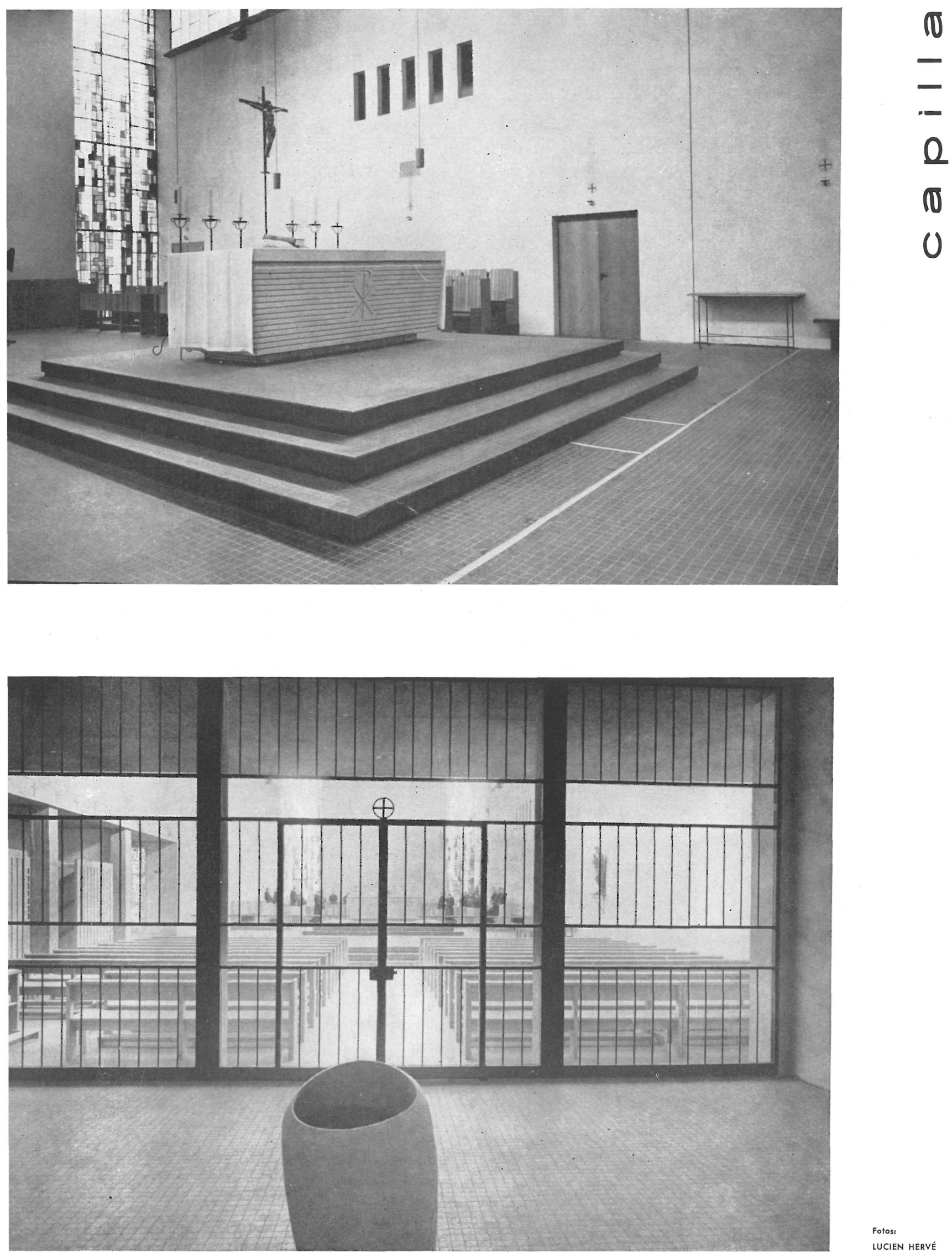

LUCIEN HERVÉ 